\title{
Informação sobre benzodiazepínicos: o que a internet nos oferece?
}

\author{
Information about benzodiazepines: what does the internet offer us?
}

Thales Brandi Ramos (https://orcid.org/0000-0002-1442-1020) ${ }^{1}$

Luciana Castilho Bokehi (https://orcid.org/0000-0003-1870-8202) ${ }^{1}$

Erika Barreto de Oliveira (https://orcid.org/0000-0002-6996-1657) ${ }^{1}$

Marcel da Silva Amorim Gomes (https://orcid.org/0000-0003-2281-8973) ${ }^{1}$

José Raphael Bokehi (https://orcid.org/0000-0003-2141-6948) ${ }^{2}$

Selma Rodrigues de Castilho (https://orcid.org/0000-0003-0272-4777) ${ }^{1}$
${ }^{1}$ Faculdade de Farmácia, Universidade Federal Fluminense (UFF). R. Mário Viana 523, Santa Rosa. 24241-000

Niterói RJ Brasil. thalesbrandi980@gmail.com

${ }^{2}$ Instituto de Computação, UFF. Niterói RJ Brasil.

\begin{abstract}
This study analysed the quality of information published on the internet regarding 4 benzodiazepines that are widely used in Brazil: alprazolam, bromazepam, clonazepam and diazepam. This choice is justified by the fact that these drugs are widely used and can generate chemical dependency, and the internet is an important source of information about them. We analysed 20 sites for each drug. More than half (56.3\%) of the sites were classified as deficient or very deficient. The most frequent problems with the sites were the absence of a description of the person responsible for the site (60\%), incomplete information (62.5\%), the absence of a contact for additional information (45\%) and the absence of the last date the site was updated (82\%). These results reinforce concerns regarding the quality of the health information published on the internet, which has already been noted in the literature, and the need to adopt minimum quality criteria for this information.
\end{abstract}

Key words Internet, Drug, Information
Resumo Este trabalho analisou a qualidade da informação veiculada na internet sobre 4 benzodiazepinicos amplamente utilizados no Brasil: alprazolam, bromazepam, clonazepam e diazepam. Essa escolha se justifica pelo fato desses medicamentos serem amplamente utilizados, poderem gerar dependência química e a internet ser importante fonte de informação sobre eles. Foram analisados 20 sites para cada medicamento. Mais da metade $(56,3 \%)$ dos sites foram classificados como deficientes ou muito deficientes. Os problemas mais frequentes foram a ausência da descrição do responsável pelo sítio (60\%), informação incompleta (62,5\%), ausência de contato para informação adicional (45\%) e da última data da atualização (82\%). Os resultados reforçam a preocupação com a qualidade da informação em saúde veiculada na internet, já apontada pela literatura, $e$ a necessidade de adoção de critérios mínimos de qualidade para esta informação.

Palavras-chave Internet, Medicamento, Informação 


\section{Introdução}

$\mathrm{O}$ advento da internet revolucionou o acesso à informação $0^{1-4} \mathrm{e}$ as relações em saúde $\mathrm{e}^{5-7}$. O acesso à Internet tem aumentado significativamente, alcançando pouco mais de quatro bilhões de usuários em 2018, somando 55,1\% da população mundial ${ }^{8}$. No Brasil, dados da PNAD contínua 2016 mostraram que, em 48,1 milhões de residências visitadas, havia utilização da Internet, o que representava $69,3 \%$ dos domicílios ${ }^{9}$.

A área da saúde desponta cada vez mais na disponibilidade de informações em sites e páginas de instituições públicas e privadas. O fácil acesso à informação auxilia no dia a dia tanto de profissionais de saúde quanto de usuários, influenciando diretamente a relação entre médico e paciente ${ }^{1,5,6}$. No Brasil, a internet já alcançou a marca de 75 milhões de acessos, levando o país a ocupar o quinto lugar na busca por informações sobre saúde nesse meio ${ }^{1}$.

Silvestre et al. ${ }^{6}$, entrevistando pacientes na faixa de 18-60 anos, em sala de espera para atendimento ambulatorial no Município de Tubarão, Santa Catarina, observaram que $50 \%$ dos entrevistados utilizavam a internet para ter acesso a informação sobre saúde e doença, com maior procura por parte de indivíduos na faixa etária entre 31-45 anos e das mulheres.

Moretti et al. ${ }^{10}$, entrevistando 1.828 indivíduos, observaram que a internet tem sido fonte de informação em saúde de grande relevância para a população, com predomínio de uso pelas mulheres que buscam informação para sua própria saúde (92\%). Para a maioria destas mulheres (86\%), a internet é uma de suas principais fontes de informação em saúde. Os autores relatam que $69 \%$ dos entrevistados reconhecem o impacto da internet no relacionamento com os médicos e que $16 \%$ e $10 \%$ deles pediram ao médico para receitar um medicamento específico ou para mudar o medicamento prescrito, respectivamente, após uma consulta online. Cinquenta e dois por cento destas pessoas afirmaram que os médicos atenderam a estas solicitações.

Sinclair et al. ${ }^{11}$, entrevistando 284 gestantes, observaram que $39 \%$ delas estavam usando algum medicamento quando se descobriram grávidas. Destas, $79 \%$ usaram a internet para buscar informação sobre a segurança destes medicamentos na gravidez.

Pereira Neto et al. ${ }^{12}$, analisando o perfil de consumo de informações on-line por jovens de uma comunidade popular, em sala de espera para a consulta médica em clínica de atenção primária vinculada ao Sistema Único de Saúde, observa- ram que apesar destes jovens pesquisarem informações sobre saúde na rede, continuam tendo o médico como principal fonte de informação.

No entanto, a qualidade da informação sobre saúde na internet ainda é um desafio em todo o mundo ${ }^{3,13,14}$. Muitas vezes, as informações sobre saúde veiculadas na internet são incorretas, incompletas ou incompreensíveis para os leitores, oferecendo riscos aos usuários ${ }^{15}$. Há diversos estudos que lidam com a avaliação e a qualificação de sites na Internet ${ }^{1,2,14,16-18}$. Um instrumento que é muito usado na avaliação da qualidade de páginas de saúde é o proposto pela Health On Net Foundation (HON), uma organização não governamental suíça. A Fundação HON concede um certificado de qualidade a páginas que atendam seu código de conduta, que possui sete princípios básicos: autoria, complementaridade, confidencialidade, atribuições, justificativas, transparência e honestidade da publicidade ${ }^{19}$.

No Brasil, vários autores têm proposto o desenvolvimento e o uso de mecanismos de avaliação da qualidade da informação em páginas de saúde ${ }^{2,15,20,21}$. O Centro de Vigilância Sanitária (CVS) do estado de São Paulo adaptou e traduziu um documento da Organização Mundial de Saúde (OMS), intitulado Guia para encontrar informações seguras, com o objetivo de fornecer ao usuário critérios de avaliação de conteúdo de sites, como nome e contato dos responsáveis, datas de publicação, identificação de financiadores e objetivos da página ${ }^{1,22}$. O Conselho Regional de Medicina do Estado de São Paulo também publicou um manual com princípios éticos para sites de medicina e saúde ${ }^{23}$.

Um modelo de avaliação alternativo foi criado por Pereira Neto et al. ${ }^{21}$, com a proposta de três dimensões de avaliação: conteúdo, usabilidade e legibilidade, subdivididas em indicadores aos quais são atribuídos pesos. Os valores somados permitem que se crie um ranking de páginas, possibilitando identificar pontos fracos e fortes de cada uma, além de apontar como está a qualidade da informação sobre o tema proposto.

Os benzodiazepínicos estão entre os medicamentos mais prescritos no mundo. Segundo dados do Sistema Nacional de Gerenciamento de Produtos Controlados, dos cinco princípios ativos de maior consumo no Brasil no período de 2007 a 2010, três são benzodiazepínicos ${ }^{24}$. Dois fármacos dessa classe integram a lista dos 100 medicamentos mais comercializados em 2017 no país ${ }^{25}$. Vários autores apontam a alta prevalência de uso destes medicamentos pela população brasileira $^{26-29}$, inclusive sem prescrição médica ${ }^{30}$. 
Neste cenário, o objetivo deste artigo é estudar a qualidade da informação na internet sobre 4 benzodiazepínicos amplamente utilizados no Brasil: alprazolam, bromazepam, clonazepam e diazepam. Essa escolha se justifica pelo fato desses medicamentos poderem gerar dependência química e a internet ser importante fonte de informação sobre eles.

\section{Metodologia}

A identificação dos sites se deu através de pesquisa no Google, principal buscador em uso no Brasil, usando a Denominação Comum Brasileira (DCB) dos medicamentos como palavra chave. A opção por usar a DCB de cada medicamento se deve ao fato desta permitir a recuperação tanto de sites que tratam o produto pelo nome comercial quanto daqueles que trabalham exclusivamente com esta denominação para se referir aos benzodiazepínicos. Para cada medicamento, foram escolhidos os 20 primeiros sítios recuperados. Foram utilizados como critérios de exclusão os sítios considerados oficiais de agências públicas e sites de venda sem fornecimento de informações sobre o produto. A exclusão dos sítios oficiais de agências públicas se deve ao fato de, em princípio, os mesmos não sofrem a influência direta de interesses comerciais ou de grupos de usuários, além de que usualmente são desenvolvidos por profissionais capacitados.

A escolha dos critérios de avaliação (Quadro 1) tomou por base o trabalho de Pereira Neto et al. ${ }^{21}$, nos aspectos conteúdo e design. Quanto ao conteúdo, foram considerados a acurácia e a abrangência da informação, o nome e os demais dados do proprietário/responsável pelas informações disponibilizadas assim como suas referências e data da última atualização. Para avaliar a acurácia, que envolve o grau de concordância existente entre a informação disponibilizada e a evidência científica disponível ${ }^{3}$, o conteúdo do sítio foi comparado aos dados técnicos disponíveis no bulário da Agência Nacional de Vigilância Sanitária ${ }^{31}$. O bulário da Anvisa foi tomado como parâmetro em razão do seu caráter oficial, uma vez que é aprovado pelo órgão regulador brasileiro. O mesmo texto foi empregado para analisar a abrangência da informação, definido por Pereira Neto et al.,21 como o grau de cobertura da informação. Foi considerada completa a informação que especificava, além das indicações, as contraindicações sobre o produto, a indicação de necessidade de acompanhamento profissional para o uso do mesmo e se utilizava, no corpo da informação, a DCB para se referir ao medicamento. Para avaliar a existência de interesses financeiros foram considerados tanto a indicação de onde adquirir o produto quanto o uso de expressões sugestivas de uso ou oferecimento de informações como dose, forma de usar etc. Quanto ao design, foi considerada a existência de distração, como animações, propagandas e presença de imagens, por exemplo, bem como a linguagem empregada (Quadro 1).

Cada site foi avaliado por dois membros da equipe, de forma independente. Caso houvesse desacordo entre as análises de qualquer indicador, um terceiro indivíduo realizava a análise, prevalecendo a opinião da maioria.

Os sites que obtiveram pontuação superior a 80 foram considerados de boa qualidade de informação, os de tiveram pontuação menor que

Quadro 1. Aspectos considerados na avaliação dos sítios.

\begin{tabular}{|c|l|c|c|c|}
\hline Aspecto & \multicolumn{1}{|c|}{ Critérios analisados } & $\mathbf{1 0}$ pts & $\mathbf{0}$ pts & Total \\
\hline \multirow{3}{*}{ Conteúdo } & Consta o responsável pelo sítio? & Sim & Não & 10 \\
\cline { 2 - 5 } & $\begin{array}{l}\text { Informação sobre o medicamento completa e de acordo com o bulário } \\
\text { da Anvisa? }\end{array}$ & Sim & Não & 10 \\
\cline { 2 - 5 } & Apresenta as contraindicações? & Sim & Não & 10 \\
\cline { 2 - 5 } & Indicação da necessidade de acompanhamento profissional? & Sim & Não & 10 \\
\cline { 2 - 5 } & Usa denominação comum brasileira (DCB)? & Sim & Não & 10 \\
\cline { 2 - 5 } & Consta a data da última atualização? & Sim & Não & 10 \\
\cline { 2 - 5 } & Existe contato para informação adicional? & Sim & Não & 10 \\
\cline { 2 - 5 } & Apresenta orientação sugestiva de uso? & Não & Sim & 10 \\
\cline { 2 - 5 } & Existe a sugestão de onde se adquirir o medicamento? & Sim & 10 \\
\hline Design & Existe algum elemento de distração? & Sim & 10 \\
\hline & Total & & 100 \\
\hline
\end{tabular}


80 e maior ou igual a 50 foram considerados regulares, os com pontuação menor que 50 e maior ou igual a 30 foram considerados deficientes e aqueles com pontuação menor que 30 , muito deficientes.

Os dados da avaliação foram armazenados em uma planilha do software Microsoft Excel 2010. Para a análise dos resultados foram empregadas as ferramentas da estatística descritiva.

Paralelamente, avaliaram-se os sites que dispunham do selo de qualidade da Fundação HON. Também foi analisada a clareza e a adequação da linguagem tomando-se por parâmetro o uso de termos técnicos que dificultam a compreensão do texto e o tipo de linguagem adotada (técnica ou leiga).

O teste Kruskal-Wallis foi empregado para avaliar a diferença de pontuação obtida pelos diferentes tipos de sites (blogs, sites de vendas e sites de discussão) e entre os diferentes medicamentos.

\section{Resultados}

No total foram analisados 80 sites (vinte para cada medicamento), dentre eles a maioria eram blogs $(40 \%)$, sites de vendas/empresas $(27,5 \%)$, sites que apresentavam bulas dos medicamentos $(21,2 \%)$ e sites de discussão $(2,5 \%)$.

Os sites analisados apresentaram menor pontuação e, portanto mais problemas, em relação à apresentação do responsável pelo sítio (60\%), informação incompleta $(62,5 \%)$, contato para informação adicional (45\%) e data da atualização (82\%) (Figura 1).

De modo geral, a presença de elementos de distração do usuário, como imagens e vídeos, foi observada na forma de anúncios em quase metade dos sites $(47,5 \%)$, sobretudo naqueles relacionados aos medicamentos bromazepam (65\%) e alprazolam (50\%). A sugestão de onde adquirir o produto foi observada em $31,2 \%$ dos sites, enquanto as contra-indicações estavam ausentes em 37,5\% deles (Tabela 1).

Para o alprazolam, $90 \%$ dos sites foram considerados regulares ou deficientes (pontuação média $=45$, moda $=40)$. Para o bromazepam, a maioria $(55 \%)$ foi considerada deficiente (pontuação média $=48,5$, moda $=40)($ Tabela 2$)$. Para o clonazepam $60 \%$ dos sites se mostraram deficientes ou muito deficientes (pontuação média = 47 , moda $=40)$ e para o diazepam, $50 \%$ foram considerados bons ou regulares e $50 \%$ deficientes $($ pontuação média $=52$, moda $=40)($ Tabela 2$)$.
A forma de adquirir o medicamento estava presente em $40 \%$ dos sites de clonazepam e diazepam, estando presente em apenas $25 \%$ e $20 \%$ dos sites de alprazolam e bromazepam, respectivamente. A necessidade de orientação profissional para consumo estava ausente em $45 \%$ dos sites do clonazepam e bromazepan, o que pode influenciar o consumo por autoindicação. A ausência de contato para informação adicional foi observada em $85 \%$ dos sites sobre o clonazepam.

Todos os sites de empresas especializadas em vendas exibiram o indicativo de "proibido venda na internet", conforme determinado pela ANVISA.

Mais da metade $(56,3 \%)$ dos sites foram classificados como deficientes ou muito deficientes. (Tabela 2). Apenas dois sites (2,5\%) possuíam o selo $\mathrm{HON}$ na época da análise. $\mathrm{O}$ primeiro foi considerado o mais bem pontuado tanto para o alprazolam quanto para o bromazepam, mas foi classificado como regular para o bromazepam e clonazepam. A informação disponibilizada no segundo site, no entanto, foi considerada deficiente para os 4 medicamentos.

A comparação da pontuação obtida pelos diferentes medicamentos não mostrou diferença estatisticamente significante a um nível de significância de $5 \%(p=0,7168)$. Também não se observou diferença estatisticamente significante na pontuação entre os diferentes tipos de site de cada um dos medicamentos: alprazolam $(\mathrm{p}=0,9933)$, bromazepam $(\mathrm{p}=0,8932)$, clonazepam $(\mathrm{p}=0,8378)$ e diazepam $(\mathrm{p}=0,6866)$. No entanto, ao se considerar o conjunto completo de sites analisados, a diferença de pontuação obtida pelos diferentes tipos de site se mostrou estatisticamente significante $(\mathrm{p}=0,00018)$, com melhor pontuação para aqueles no formato de bulas dos medicamentos. Não se observou diferença estatisticamente significante entre os resultados dos sites de empresas e blogs entre si $(\mathrm{p}=1)$, ambos inferiores aos sites de bulas.

Para os sites que falavam do diazepam, a principal fonte de contato adicional foi o telefone $(50 \%)$, seguida de mecanismos de perguntas e respostas $(37,5 \%)$ e de e-mail $(18,75 \%)$. Para o bromazepam, os mecanismos de perguntas e respostas representaram a forma de contato adicional em $45 \%$ dos sites, enquanto o telefone representou 55\% das opções de contato. Com o clonazepam, em todos os 3 sites que apresentaram forma de contato adicional o veículo foi o telefone. Com o alprazolam, apenas $45 \%$ dos sites apresentavam forma adicional de contato: 55\% usavam perguntas e respostas, $22,5 \%$ o telefone e o email cada. 


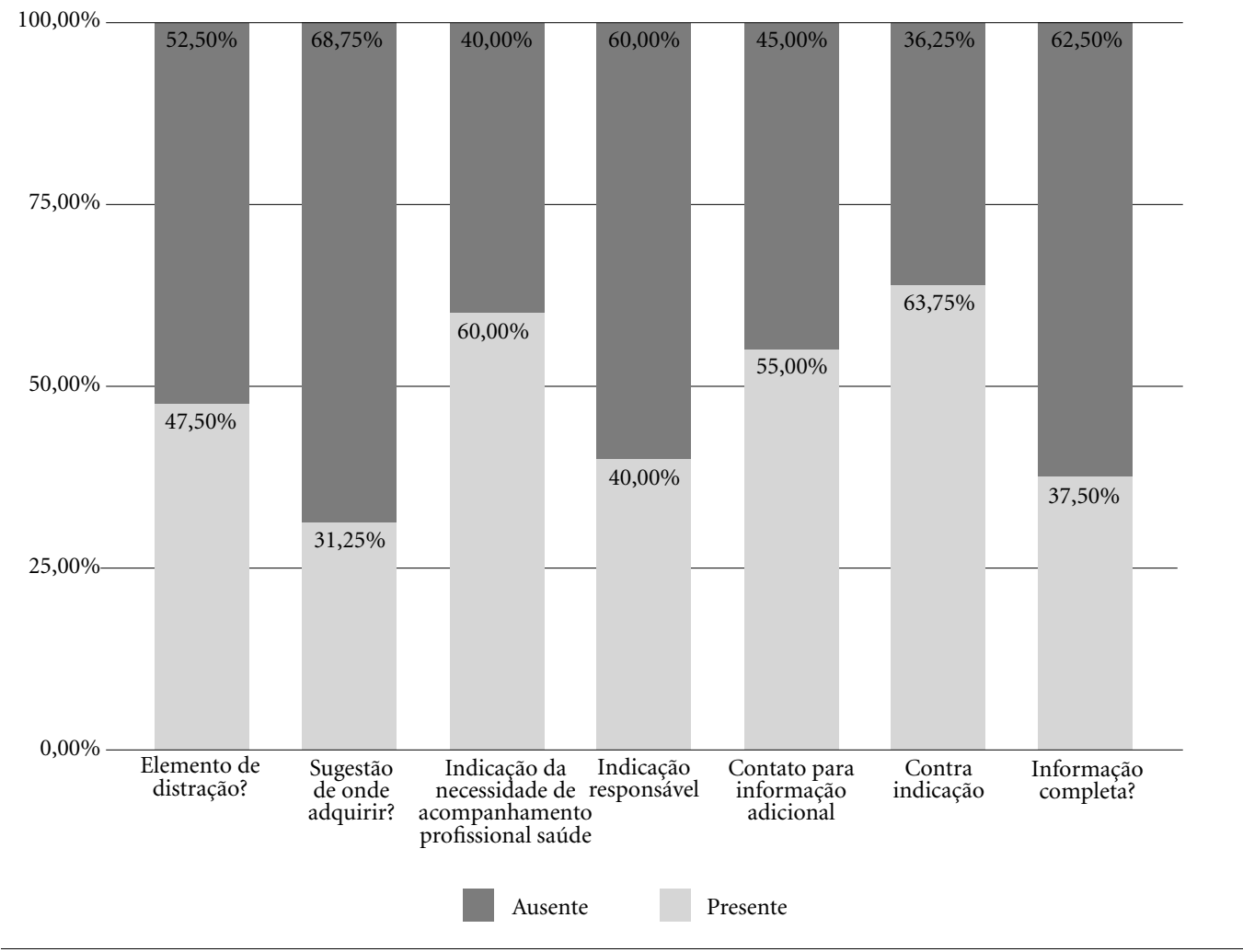

Figura 1. Desempenho global dos sites analisados $(\mathrm{N}=80), 2018$.

Tabela 1. Percentual de atendimento aos indicadores dos sites analisados para cada medicamento, $\mathrm{N}=80$, Niterói, 2018.

\begin{tabular}{|c|c|c|c|c|c|c|c|c|c|}
\hline & \multirow{3}{*}{ Aspecto } & \multicolumn{8}{|c|}{ Percentual observado } \\
\hline & & \multicolumn{2}{|c|}{ Alprazolam } & \multicolumn{2}{|c|}{ Bromazepam } & \multicolumn{2}{|c|}{ Clonazepam } & \multicolumn{2}{|c|}{ Diazepam } \\
\hline & & $\%$ & $\mathbf{N}$ & $\%$ & $\mathbf{N}$ & $\%$ & $\mathbf{N}$ & $\%$ & $\mathbf{N}$ \\
\hline \multirow[t]{9}{*}{ Conteúdo } & Consta o responsável pelo sítio? & 35 & 7 & 35 & 7 & 40 & 8 & 40 & 8 \\
\hline & $\begin{array}{l}\text { Informação sobre o } \\
\text { medicamento completa e de } \\
\text { acordo com o bulário da Anvisa? }\end{array}$ & 35 & 7 & 35 & 7 & 40 & 8 & 40 & 8 \\
\hline & Apresenta as contraindicações? & 55 & 11 & 65 & 13 & 65 & 13 & 65 & 13 \\
\hline & $\begin{array}{l}\text { Indicação da necessidade de } \\
\text { acompanhamento profissional? }\end{array}$ & 70 & 14 & 55 & 11 & 55 & 11 & 60 & 12 \\
\hline & $\begin{array}{l}\text { Usa denominação comum } \\
\text { brasileira? }\end{array}$ & $100 *$ & 20 & $95^{\star *}$ & 19 & $90^{* *}$ & 18 & $90^{* * *}$ & 18 \\
\hline & $\begin{array}{l}\text { Consta a data da última } \\
\text { atualização? }\end{array}$ & 10 & 2 & 10 & 2 & 25 & 5 & 25 & 5 \\
\hline & $\begin{array}{l}\text { Existe contato para informação } \\
\text { adicional? }\end{array}$ & 45 & 9 & 60 & 12 & 55 & 11 & 60 & 12 \\
\hline & $\begin{array}{l}\text { Apresenta orientação sugestiva } \\
\text { de uso? }\end{array}$ & 25 & 5 & 20 & 4 & 40 & 8 & 40 & 8 \\
\hline & $\begin{array}{l}\text { Existe a sugestão de onde se } \\
\text { adquirir o medicamento? }\end{array}$ & 25 & 5 & 20 & 4 & 40 & 8 & 40 & 8 \\
\hline Design & $\begin{array}{l}\text { Existe algum elemento de } \\
\text { distração? }\end{array}$ & 50 & 10 & 65 & 13 & 25 & 5 & 40 & 8 \\
\hline
\end{tabular}

* dois sites usavam tanto a DCB quanto o nome comercial do produto. ${ }^{* \star}$ dois sites usavam tanto a DCB quanto o nome comercial do produto. ${ }^{* * *}$ quatro sites usavam tanto a DCB quanto o nome comercial do produto. 
Tabela 2. Distribuição das classificações dos 20 sites por medicamento.

\begin{tabular}{|c|c|c|c|c|c|c|c|c|}
\hline \multirow{2}{*}{ Medicamento/ Classificação site } & \multicolumn{2}{|c|}{ Bom } & \multicolumn{2}{|c|}{ Regular } & \multicolumn{2}{|c|}{ Deficiente } & \multicolumn{2}{|c|}{$\begin{array}{c}\text { Muito } \\
\text { deficiente }\end{array}$} \\
\hline & $\mathbf{N}$ & $\%$ & $\mathbf{N}$ & $\%$ & $\mathbf{N}$ & $\%$ & $\mathbf{N}$ & $\%$ \\
\hline Alprazolam & 0 & 0 & 9 & 45 & 9 & 45 & 2 & 10 \\
\hline Bromazepam & 3 & 15 & 5 & 25 & 11 & 55 & 1 & 5 \\
\hline Clonazepam & 2 & 10 & 6 & 30 & 10 & 50 & 2 & 10 \\
\hline Diazepam & 4 & 20 & 6 & 30 & 10 & 50 & 0 & 0 \\
\hline Total & 9 & 11,25 & 26 & 32,5 & 40 & 50 & 5 & 6,25 \\
\hline
\end{tabular}

$\mathrm{O}$ uso de linguagem leiga foi observado em $55 \%$ dos sites envolvendo o alprazolam e o bromazepam, $45 \%$ dos sites de diazepam e em $40 \%$ dos sites do clonazepam. O desbalanceamento entre as indicações de uso e a apresentação das contraindicações e cuidados foi observado em $36,5 \%$ de todos os sites analisados.

\section{Discussão}

Dentre os 80 sites analisados, $56,3 \%$ foram classificados como deficientes ou muito deficientes. Este resultado está de acordo com o encontrado por Pereira Neto et al. ${ }^{21}$, que ao avaliarem 8 sites de ONG relacionados ao HIV/AIDS, observaram que apenas um apresentava conteúdo concordante com o esperado em pelo menos $50 \%$. Resultado semelhante foi observado por Neumark et al. ${ }^{14}$ analisando 29 sites sobre contraceptivos em língua hebraica com base nos códigos HON. A nota média dos sites para acurácia foi de 50,9\%.

Paolucci et al. ${ }^{15}$, avaliando a qualidade da informação sobre tuberculose em sites brasileiros viram que nenhum site obteve mais que $65 \%$ de conformidade com os critérios de qualidade adotados na avaliação. Del Giglio et al. ${ }^{32}$, avaliando a qualidade da informação relacionada a Diabete Mellitus, Hipertensão Arterial Sistêmica e Infarto Agudo do Miocárdio, também em sites nacionais, observaram que as informações eram frequentemente inadequadas e insuficientes.

Yoon et al. ${ }^{18}$, analisando sites coreanos sobre doença inflamatória intestinal, concluíram que a qualidade da informação não é ideal e varia significativamente tanto em relação ao tipo de site, mas também com relação à ferramenta de busca utilizada. Os autores também observaram que a qualidade da informação não está relacionada à posição na qual o site aparece nos resultados da busca.
A ausência do responsável pelo conteúdo $(60 \%)$ e da data de atualização $(82 \%)$ na maioria dos sites analisados afeta diretamente a confiabilidade destes, como apontado por Newmark et al. ${ }^{14}$, Mendonça e Pereira Neto ${ }^{2}$ e Molino e Melo ${ }^{33}$. Além disso, inviabilizam a responsabilização sobre a informação prestada. Estes resultados estão de acordo com outros trabalhos ${ }^{15,34,35}$. Por outro lado, Hirata et al. ${ }^{36}$ identificaram a descrição do responsável em 79,3\% dos 4 sites brasileiros sobre coronariopatia analisados.

A disponibilização de informações incompletas observada em $62,5 \%$ dos sites está de acordo com a literatura ${ }^{1,14,35,37-39}$ e compromete a possibilidade de uma análise adequada do usuário sobre a segurança e a oportunidade de utilização do produto ou do problema de saúde tratado pelo site. O desbalanceamento na informação, também apontado por vários autores ${ }^{1,35,38}$, com destaque para os benefícios e atenuação das contraindicações, é outro aspecto que pode expor o usuário a risco ao induzi-lo ao consumo de produtos desnecessários ou inadequados à sua saúde. Esta mesma estratégia é observada em peças publicitárias sobre medicamentos como, por exemplo, propagandas de medicamentos veiculadas em rádios ${ }^{40}$ e dispostas em farmácias e drogarias $^{41}$. Estratégia semelhante foi observada em artigos de jornalismo científico de uma revista de grande circulação no Brasil ${ }^{13}$.

A preocupação com a qualidade da informação disponibilizada especificamente sobre medicamentos psicoativos, como os benzodiazepínicos, em peças publicitárias também foi apontada por Mastroianni et al. ${ }^{38}$. Os autores apontam que as peças apresentavam informações incompletas, resumidas, inconsistentes e diferentes dos estudos citados no texto, sempre favoráveis à indicação terapêutica, eficácia, segurança e custo dos produtos. 
A internet vai continuar a crescer como provedor de informação sobre saúde, mas o meio pelo qual esta informação chega da fonte ao paciente não deveria comprometer características como a acurácia, credibilidade, qualidade e compreensibilidade da informação ${ }^{42}$. Neste contexto, o engajamento dos responsáveis pelos sites que fornecem informação em saúde a códigos de conduta ou a padrões de qualidade é importante ${ }^{43-45}$. Varady et al. $^{44}$, analisando sites contendo informação sobre osteoartrite em vários países observaram que os sites com certificação $\mathrm{HON}$ ou usando o instrumento DISCERN, empregado para avaliar a qualidade da informação escrita para consumidores sobre escolhas de tratamento ${ }^{46}$, obtiveram escores de avaliação significativamente superiores aos sem esta certificação. Resultado semelhante é apontado por outros estudos ${ }^{44-46}$,

Neste sentido, o baixo número de sites sobre os benzodiazepínicos com a certificação HON (2,5\%) é preocupante. O percentual de sites com certificação HON variou bastante entre diferentes estudos ${ }^{43,47-50}$, tendo sido encontrados valores entre quatro (4) e $60 \%$ de sites certificados. Igualmente relevante é o fato de que, mesmo nos sites que apresentavam a certificação HON foram observados problemas de qualidade da informação. Este resultado está de acordo com o observado por Grohol et al. ${ }^{50}$.

A diferença estatisticamente significativa na avaliação dos 80 sites analisados em função do tipo de site está de acordo com o observado por outros autores ${ }^{18,50}$. Yoon et al. ${ }^{18}$ observaram superioridade da qualidade dos sites institucionais. Já os sites de suporte (mantidos por grupos de apoio, usuários etc) apresentou o menor escore na avaliação de qualidade. Grohol et al. ${ }^{50}$ observaram superioridade na qualidade dos sites com o selo HONCode e sem interesses comerciais. A variação em relação à ferramenta de busca apontada por estes autores, no entanto, não foi analisada neste trabalho.

Outro aspecto relevante é o fato dos benzodiazepínicos serem apontados, por alguns autores $^{51-53}$, como os principais medicamentos envolvidos em intoxicações exógenas no Bra- sil. É razoável supor que a facilidade de acesso à informação sobre estes produtos na internet e a baixa qualidade desta informação podem ser fatores que contribuem para o uso irracional destes produtos. Soma-se ainda o fato de que, no Brasil, mesmo medicamentos que requerem a apresentação de prescrição médica são adquiridos com facilidade no mercado ${ }^{54,55}$. Diversos autores ${ }^{30,54-56}$ apontam o uso indiscriminado, inclusive por automedicação, dos benzodiazepínicos, e suas consequências para a saúde da população.

\section{Conclusão}

Apesar do número reduzido de sites analisados, de terem sido analisados apenas sites na língua portuguesa e recuperados por uma única ferramenta de busca, bem como o uso do bulário da Anvisa como parâmetro de comparação da informação sobre os produtos, principais limitações deste estudo, os resultados mostram a baixa qualidade da informação disponibilizada na internet sobre os medicamentos benzodiazepínicos. Desta forma, reforçam a discussão mundial sobre a necessidade de regulamentação dos conteúdos relacionados à saúde disponíveis na internet.

O baixo percentual de sites que dispõem de um selo de qualidade, como o HON, por exemplo, mostra a baixa preocupação dos desenvolvedores destas páginas com a possibilidade de oferecer um parâmetro confiável de avaliação do conteúdo do site para os usuários. Isto é reforçado pela ausência de informações básicas como a identificação dos responsáveis pelo conteúdo disponível, as referências utilizadas e a data da atualização do conteúdo, todas relevantes para a confiabilidade das informações prestadas.

Desta forma, a regulamentação da informação presente nos sites e a aplicação de selos de qualidade no Brasil se mostram cruciais para que o usuário saiba quais informações podem ser confiáveis, evitando assim o uso irracional de medicamentos e os graves problemas de saúde que podem decorrer do uso destes por ausência ou baixa qualidade da informação disponibilizada. 


\section{Colaboradores}

SR Castilho participou da definição da metodologia, da análise dos resultados e da redação do texto. TB Ramos, LC Bokehi, EB Oliveira e MSA Gomes participaram da discussão da metodologia, coletaram os dados, participaram da análise dos dados e da redação do texto. JR Bokehi participou da discussão da metodologia, da análise dos dados e da revisão do texto.

\section{Referências}

1. Oliveira F, Bertollo-Golloni EM, PavarinoEC. A Internet como fonte de Informação em Saúde. J Health Inform 2013; 5(3):98-102.

2. Mendonça APB, Pereira Neto A. Critérios de avaliação da qualidade da informação em sites de saúde: uma proposta. RECIIS 2015; 9(1):1-15.

3. Pereira Neto, Faria A, Paolucci R, Daumas RP, Souza RV. Avaliação participativa da qualidade da informação de saúde na internet: o caso de sites de dengue. Cien Saude Colet 2017; 22(6):1955-1968.

4. Biggs TC, Jayakody N, Best K, King EV. Quality of online otolaryngology health information. J Laryngol Otol 2018; 132(6):560-563.

5. Garbin HBR, Pereira Neto AF, Guilam MCR. The internet, expert patients and medical practice: an analysis of the literature. Interface (Botucatu) 2008; 12(26):579-588.

6. Silvestre JCC, Rocha PAC, Silvestre BC, Cabral RV, Trevisol FS. Uso da internet pelos pacientes como fonte de informação em saúde e a sua influência na relação medico-paciente. Revista da AMRIGS 2012; 56(2):149-155.

7. Garbin HBR, Guilam MCR, Pereira Neto AF. Internet na promoção da saúde: um instrumento para o desenvolvimento de habilidades pessoais e sociais. Physis 2012; 22(1):347-363.

8. Internet World Stats [Internet]. Miniwatts Marketing Group. Population Statistics [about 1 screen]. [cited 2018 Sep 22]. Available from: https://www.internetworldstats.com/stats.htm

9. Instituto Brasileiro de Geografia e Estatística (IBGE). PNAD Contínua TIC 2016: IBGE notícias. 2018. Available from: https://agenciadenoticias.ibge.gov.br/ agencia-sala-de-imprensa/2013-agencia-de-noticias/ releases/20073-pnad-continua-tic-2016-94-2-das-pessoas-que-utilizaram-a-internet-o-fizeram-para-trocarmensagens

10. Moretti FA, Oliveira VE, Silva EMK. Acesso a informações de saúde na internet: uma questão de saúde pública? Rev Assoc Med Bras 2012; 58(6):650-658.

11. Sinclair M, Lagan BM, Dolk H, McCullough JEM. An assessment of pregnant women's knowledge and use of the Internet for medication safety information and purchase. J Adv Nurs 2017; 74(1):137-147.

12. Pereira Neto AF, Barbosa L, Muci S. Internet, geração Y e saúde: um estudo nas comunidades de Manguinhos (RJ). Comun. \& Inf 2016; 19(1):20-36.

13. Vaz P, Portugal DB. A nova "boa-nova": marketing de medicamentos e jornalismo científico nas páginas da revista brasileira Veja. Comun Mídia Consumo 2012; 9(26):37-60.

14. Neumark Y, Flum L, Lopez-Quintero C, Shtarkshall R. Quality of online health information about oral contraceptives from Hebrew-language websites. Isr J Health Policy Res 2012; 1(1):38.

15. Paolucci R, Pereira Neto A, Luzia R. Avaliação da qualidade da informação em sites de tuberculose: análise de uma experiência participativa. Saúde debate 2017; 41(n. esp.):84-100.

16. Eysenbach G, Powell J, Kuss O, Sá ER. Empirical studies assessing the quality of health information for consumers on the World Wide Web. A systematic review. JAMA 2002; 287(20):2691-2700. 
17. Lopes IL. Novos paradigmas para avaliação da qualidade da informação em saúde recuperada na Web. $C i$ Inf 2004; 33(1):81-90.

18. Yoon JS, Lee SJ, Kim ES, Kim SK, Jung MK, Lee HS, Kwon YH, Nam SY, Jeon SW, Jin S, Lee JS, Yeo SJ. Quality of information on the Internet for Korean patients with inflammatory bowel disease. Korean J Intern Med 2018; 34(6):1215-1222.

19. Health on the Net Foundation (HON) [Internet]. Código de conduta para sites web em Medicina e Saúde (HONcode). [cited 2018 Sep 24]. Available from: http://www.hon.ch/HONcode/Portuguese

20. Santos V, Portal MM, Ferigolo M, Dantas DCM, Barros HMT, Trindade CS. Sites sobre drogas de abuso: recursos para avaliação. Trab. educ. saúde 2010; 8(3):575-585.

21. Pereira Neto AF, Santos EM, Cruz MM, Torres RMC. Avaliação de sites de saúde em questão: a Aids nos sites brasileiros de Organizações Não Governamentais (ONG) de Lésbicas, Gays, Bissexuais, Travestis e Transexuais (LGBT). Rev Electron Comun Inf Inov Saude 2013; 7(1).

22. Lopes IL. Novos paradigmas para avaliação da qualidade da informação em saúde recuperada na Web. Ci. Inf. 2004; 33(1):81-90.

23. Conselho Regional de Medicina do Estado de São Paulo (CREMESP) [Internet]. Manual de princípios éticos para sites de medicina e saúde na Internet. [cited 2018 Sep 25]. Available from: http://www.saudeinformacoes.com.br/institucional_cremesp.asp

24. Brasil. Agência Nacional de Vigilância Sanitária (Anvisa). Boletim de Farmacoepidemiologia do SNGPC 2011; 2(1).

25. Brasil. Agência Nacional de Vigilância Sanitária (Anvisa). Anuário estatístico do mercado farmacêutico 2016. Brasília: Anvisa; 2017.

26. Firmino KF, Abreu MHNG, Perini E, Magalhães SMS Utilização de benzodiazepínicos no Serviço Municipal de Saúde de Coronel Fabriciano, Minas Gerais. Cien Saude Colet 2012; 17(1):157-166.

27. Lopes AP. Ansiedade e consumo de substâncias psicoativas em adolescentes. [dissertação]. São Bernardo do Campo: Faculdade de Saúde da Universidade Metodista de São Paulo; 2011.

28. Lopes AP, Rezende MM. Ansiedade e consumo de substâncias psicoativas em adolescentes. Estud psicol 2013; 30(1):49-56

29. Carlini EA, Noto AR, Sanchez ZM, Carlini CMA, Locatelli DP, Abeid LR, Amato TC, Opaleye ES, Tondowski CS, Moura YG, organizadores. VI Levantamento Nacional sobre o Consumo de Drogas Psicotrópicas entre Estudantes do Ensino Fundamental e Médio das Redes Pública e Privada de Ensino nas 27 Capitais Brasileiras - 2010. São Paulo, Brasília: Centro Brasileiro de Informações sobre Drogas Psicotrópicas (CEBRID), Universidade Federal de São Paulo (UNIFESP), Secretaria Nacional de Políticas sobre Drogas (SENAD); 2010
30. Galduróz JCF, Noto AR, Fonseca AM, Carlini EA. V Levantamento sobre o consumo de drogas psicotrópicas entre estudantes do ensino fundamental e médio da rede pública de ensino nas 27 capitais brasileiras - 2004 . São Paulo, Brasília: Centro Brasileiro de Informações sobre Drogas Psicotrópicas (CEBRID), Universidade Federal de São Paulo (UNIFESP), Secretaria Nacional de Políticas sobre Drogas (SENAD); 2005.

31. Brasil. Agência Nacional de Vigilância Sanitária (Anvisa). Bulário Eletrônico. [cited 2018 Sep 25]. Availabe at: http://www.anvisa.gov.br/datavisa/fila_bula/index. asp

32. Del Giglio A, Abdala B, Ogawal C, Amado D, Carter D, Gomieiro F, Salama F, Shiroma M, Del Giglio A. Qualidade da informação da internet disponível para pacientes em páginas em português. Rev Assoc Med Bras 2012; 58(6):645-649.

33. Molino CGRC, Melo DO. A criação de sítio para disseminar informações sobre medicamentos no SUS: um relato de experiência. Rev Eletron Comun Inf Inov Saúde 2017; 11(1):1-9.

34. Gondim AP, Weyne DP, Ferreira BS.Qualidade das informações de saúde e medicamentos nos sítios brasileiros. Einstein 2012; 10(3):335-341

35. Silva EV, Castro LLC, Cymrot R. Informação sobre o tratamento farmacológico da obesidade em sítios da Internet: avaliação da qualidade. Rev Tempus Actas Saúde Colet 2010; 4(3):95-111.

36. Hirata DM, Queiroz NR, Souza RC, Oliveira LB, Martins WA. Qualidade da Informação na Internet sobre Coronariopatia. Rev Bras Cardiol 2010; 23(1):39-46.

37. Parr O, Dunmall K. An evaluation of online information available for women with breast implants aged 47-73 who have been invited to attend the NHS Breast Screening Programme. Radiography (Lond) 2018; 24(4):315-327

38. Mastroianni PC, Notol AR, Galduróz JCF. Propagandas de medicamentos psicoativos: análise das informações científicas. Rev Saude Publica 2008; 42(3):529535.

39. Hernández-García I, Giménez-Júlvez T. Evaluación de la información sanitaria disponible en internet sobre las recomendaciones de vacunación frente al Meningococo B. Rev Esp Salud Publica 2018; 92:e201805017.

40. Batista AM, Carvalho MCRD. Avaliação da propaganda de medicamentos veiculada em emissoras de rádio. Cien Saude Colet 2013; 18(2):553-561.

41. Silva PS, Rangel BCC, Castilho SR. Avaliação da propaganda de medicamentos isentos de prescrição em farmácias comunitárias do município de Niterói (RJ, Brasil). R Dir Sanit 2017; 18(3):77-93.

42. Hirsch M, Aggarwal S, Barker C, Davis CJ, Duffy JMN. Googling endometriosis: a systematic review of information available on the Internet. Am J Obstet Gynecol 2017; 216(5):451-458.

43. Falcão AEJ, Mancini F, Teixeira FO, Sousa FS, Hummel AD, Aureliano KC, Costa TM, Sigulem D, Pisa IT. Análise da percepção de usuários sobre a qualidade de websites em saúde comparada com os critérios de adequação da HON. J Health Inform 2011; 3(3):103-8. 
44. Varady NH, Dee EC, Katz JN. International assessment on quality and content of internet information on osteoarthritis. Osteoarthritis Cartilage 2018; 26(8):1017-1026.

45. Ottenhoff JSE, Kortlever JTP, Teunis T, Ring DJ. Factors Associated With Quality of Online Information on Trapeziometacarpal Arthritis. J Hand Surg Am 2018; 43(10):889-896.

46. Charnock D, Shepperd S, Needham G, Gann R. DISCERN: an instrument for judging the quality of written consumer health information on treatment choices. J Epidemiol Community Health 1999; 53(2):105-111.

47. Fast AM, Deibert CM, Boyer C, Hruby GW, McKierman JM. Partial nephrectomy online: a preliminary evaluation of the quality of health information on the Internet. BJU Int 2012; 110 (11 Pt B):E765-769.

48. Raj S, Sharma VL, Singh AJ, Goel S. Evaluation of Quality and Readability of Health Information Websites Identified through India's Major Search Engines. Adv Prev Med 2016; 2016:4815285.

49. Fischer JH, O'Connor D, Flexman AM, Shapera S, Ryerson CJ. Accuracy and Reliability of Internet Resources for Information on Idiopathic Pulmonary Fibrosis. Am J Respir Crit Care Med 2016; 194(2):218225.

50. Grohol JM, Slimowicz J, Granda R. The quality of mental health information commonly searched for on the internet. Cyberpsychol Behav Soc Netw 2014; 17(4):216-221.

51. Costa AO, Alonzo HGA. Casos de exposição e intoxicações por medicamentos registrados em um Centro de Intoxicações do interior do estado de São Paulo. Rev Bras Pesq Saúde 2015; 17(2):52-60.
52. Monte BS, Nunes MST, Nunes MDS, Mendes CMM. Estudo epidemiológico das intoxicações por medicamentos registrados pelo centro de informações toxicológicas do Piauí: 2007 a 2012. R Interd 2016; 9(3):96-104.

53. Silva RLF, Sampaio PR, Estephanin VV, Leite ICG, Bonfante HL. Perfil epidemiológico das intoxicações exógenas na cidade de Juiz de Fora - MG. HU Revista 2017; 43(2) 149-154.

54. Silva IM, Catrib AMF, Matos VC, Gondim APS. Automedicação na adolescência: um desafio para a educação em saúde. Cien Saude Colet 2011; 16(Supl. 1):1651-1660.

55. Castro GLG, Mendes CMM, Pedrini ACR, Gaspar DSM, Sousa FCF. Uso de Benzodiazepínicos como automedicação: consequências do uso abusivo, dependência, farmacovigilância e farmacoepidemiologia. $R$ Interd 2013; 6(1):112-123.

56. Silva EG, Fernandes DR, Terra Júnior AT. Uma abordagem ao uso indiscriminado de medicamentos benzodiazepínicos. Rev Cient FAEMA 2018; 9:610-614.

Artigo apresentado em 05/11/2018

Aprovado em 22/04/2019

Versão final apresentada em 24/04/2019 